\title{
Emerging Rickettsial Diseases: an Analysis of Undifferentiated Acute Febrile Illness Cases from a Tertiary Care Hospital in New Delhi
}

\author{
Garima Gautam*, Manoj Jais, Anupam Prakash and Harish K. Pemde \\ Department of Microbiology, First floor, Lal building, Lady Hardinge Medical College, \\ Connaught Place, New Delhi 110001, India \\ *Corresponding author
}

K e y w o r d s
Rickettsial diseases,
Weil Felix Test
(WFT), Scrub
typhus, Acute
febrile illness,
Eschar
Article Info
Accepted:
07 February 2019
Available Online:
10 March 2019

\section{Introduction}

Rickettsial infections are being increasingly recognized as a cause of acute febrile illnesses and patients presenting with suggestive clinical features should be considered a distinct possibility. ${ }^{1}$ It includes three major groups: Scrub typhus, spotted fever group and the typhus group.

\section{A B S T R A C T}

Rickettsial diseases are an important cause of undifferentiated acute febrile illness. The lack of classical manifestations can make their diagnosis difficult. Even though the diseases are endemic in India, they are less reported from New Delhi and surrounding regions. Aim is to estimate the seropositivity of Rickettsial infections in cases of undifferentiated acute febrile illness from a tertiary care hospital in New Delhi and to analyze their clinical profile. This prospective observational study was conducted from November 2016 to March 2018. Samples were first screened for Typhoid, Dengue, Chikungunya and Malaria. Samples seronegative for these diseases were tested further by Weil- Felix test (WFT) and clinical findings were recorded on a pre designed proforma. Data was analyzed using statistical software SPSS version 21. A total of 370 seronegative samples were tested. Out of 370 cases, $12.4 \%$ (46) cases were positive by Weil- Felix reaction and most of the cases showed titre between $\geq 1: 80-\geq 1: 160$. Of the positive cases, $7.29 \%$ were positive for scrub typhus (OXK) and $3.78 \%$ cases were positive for tick typhus (OX2) whereas only $1.35 \%$ cases were found positive for typhus group (OX19). The most common presentation was fever with headache and pulmonary manifestations or rashes. The most common laboratory findings were increase in AST/ALT, anaemia and thrombocytopenia. The findings of our study indicate significant presence of rickettsial diseases in cases of undifferentiated febrile illness in Delhi population. 
typhus has been reported in Himachal Pradesh in September $2016 .^{3}$

Diagnosis and surveillance of these diseases can be challenging. Indirect immunoperoxidase assay (IPA) and immunofluorescence assay (IFA) are considered gold standards but are available only in limited laboratories. ${ }^{4}$ However, simple, economical Weil- Felix test (WFT) as initial investigation can guide a clinician in instituting appropriate treatment. In rickettsial infections there is development of antibodies after 5-10 days that agglutinate certain strains of non motile Proteus organisms, i.e. $P$ vulgaris $0 \mathrm{X} 19$ and $0 \mathrm{X} 2$ and $P$. mirabilis OXK. The sharing of these antigens between Rickettsia and Proteus is the basis of this heterophile antibody test.

The objective of the study was to estimate the seropositivity of rickettsial infections in cases of undifferentiated acute febrile illness from a tertiary care hospital in New Delhi and to analyze the clinical profile of the patients seropositive for rickettsial infections.

\section{Materials and Methods}

This prospective observational study was conducted at Department of Microbiology, Lady Hardinge Medical College and associated Hospitals, New Delhi from November 2016 to March 2018.

Blood samples of the patients having fever submitted to Microbiology Department, Lady Hardinge Medical College, were taken for serological testing with informed consent.

Patients with history of fever for more than one week, from 5 to 60 years of age and seronegative for Typhoid, Dengue, Chikungunya and Malaria were included in the study. Samples of HIV positive patients or patients with any other immunodeficiency condition or on cancer chemotherapy were excluded.

Blood samples were collected and centrifuged at 1000 rpm for 5 minutes to obtain serum. Serum was collected by calibrated micropipette into separate sterile vials and stored at $2-8^{0} \mathrm{C}$ till testing was complete.

Samples were first screened for Typhoid, Dengue, Chikungunya and Malaria. For typhoid, Widal tube agglutination test was employed. Any sample with significant titre for $\mathrm{T}_{\mathrm{O}}, \mathrm{T}_{\mathrm{H}}, \mathrm{A}_{\mathrm{H}}$ or $\mathrm{B}_{\mathrm{H}}$ (i.e. $\geq 1: 160$ ) was rejected. For dengue and chikungunya, MAC ELISA and IgM ELISA were employed, respectively. Samples with value more that calculated OD cut-off were rejected. Lastly, rapid pan malaria ICT was used to reject malaria positive samples.

A total of 370 samples seronegative for typhoid, dengue, chikungunya and malaria were included in the study. Clinical findings were recorded on a pre designed proforma.

Weil Felix test was performed by using microtitre plate agglutination method as previously described. ${ }^{5}$ Antigens Proteus vulgaris OX2, $P . \quad$ vulgaris OX19 and $P$. mirabilis $\mathrm{OXK}$ were obtained from Central Research Institute (CRI), Kasauli, India. WFT was performed initially using doubling dilutions from 1:40 to 1:320. Sera having titres more than 320 were further screened till end titre dilution. The cut off titre for Delhi population was taken as 1:80. ${ }^{6}$

\section{Data analysis}

Data were collected, compiled and analyzed in Excel sheet. The seropositivity of the diseases and their clinical features and laboratory findings were expressed as a percentage. Data was analyzed using statistical software SPSS version 21. 


\section{Results and Discussion}

A total of 370 samples were tested out of which, 142 were males and 228 were female.

Samples were taken from patients aged 5 to 60 years; the mean age was 32.18 years (Figure 1).

Chart 1 shows distribution of patients according to the geographical area. 90.2\% patients (334) were from New Delhi population whereas 25 patients were from Utter Pradesh. Minority of patients were from Haryana (1.9\%), Punjab (0.8\%) and Bihar $(0.5 \%)$.

Out of 370 cases, 12.4\% (46) cases were positive by Weil- Felix reaction for presence of rickettsial infections. Of the positive cases, $7.29 \%$ (27) were positive for OXK (scrub typhus) and $3.78 \%$ (14) cases were positive for OX2 (spotted fever group) whereas only $1.35 \%$ (5) cases were found positive for OX19 (typhus group) (Chart 2).

Out of total 46 Weil Felix positive cases, most of the cases showed titre between $\geq 1: 80$ $\geq 1: 160$. Out of 27 OXK positive cases, $51.9 \%$ cases showed titre of $\geq 1: 80,33.3 \%$ cases showed $\geq 1: 160$ titre and $11.1 \%$ cases showed $\geq 1: 320$. Highest titre achieved was $\geq 1: 640$ showed by $3.7 \%$ cases. Out of OX2 positive cases, $42.9 \%$ cases showed titre of $\geq 1: 80$, $35.8 \%$ cases showed $\geq 1: 160$ titre and $14.2 \%$ cases showed $\geq 1: 320$. Highest titre achieved was again $\geq 1$ : 640 showed by $7.1 \%$ cases only. Out of OX19 positive cases, $20 \%$ showed titre of $\geq 1: 80$ where as rest of the showed titre of $\geq 1: 160$ (Table 1 ).

Out of 27 scrub typhus positive patients, the duration of fever at the time of sample collection varied from 7- 16 days. Maximum patients presented within 7-8 days of fever, $77.8 \%, 60 \%$ and $64.2 \%$ cases of OXK, OX19 and OX2 respectively. Prolonged history of fever (13-16 days) was seen in $11.1 \%, 20 \%$ and $7.1 \%$ cases of OXK, OX19 and OX2 respectively (Figure 2).

Figure 3 represents frequency and distribution of clinical features among Weil Felix positive cases. Among OXK positive cases, rash was seen in $37 \%$ cases (10/27). Headache and pulmonary manifestations were the most common symptoms, seen in $62.9 \%$ cases (17). Eschar was seen in $7.4 \%$ (2) cases. Other common symptoms seen were nausea vomiting (37\%) and Icterus (18.5\%) whereas haemorrhage was seen only in $18.5 \%$ cases. None of the patients presented with behaviour changes. Among OX19 and OX2 positive cases, the most common symptom was headache $(100 \%)$ followed by pulmonary manifestations (80\%). Rash was seen in $60 \%$ of OX19 cases whereas in 21\% of OX2 cases. Nausea/vomiting and icterus were seen in $60 \%$ and $20 \%$ cases of OX19 respectively as compared to $28 \%$ and $7 \%$ in OX2 cases respectively.

Figure 4 shows the most common laboratory finding was increase in AST/ALT (70\%) followed by anaemia (66.4\%) and leucocytosis (37\%) among OXK positive cases. Thrombocytopenia was seen in $26 \%$ cases. Among cases positive for OX19, common laboratory finding was anaemia $(60 \%)$, increase in AST/ALT $(60 \%)$ and thrombocytopenia (60\%) followed by leucocytosis (40\%). In OX2 positive cases as well, increase in AST/ALT (71\%) was the most common finding followed by anaemia $(50 \%)$ and thrombocytosis (50\%). Leucocytosis was rare finding seen only in $7 \%$ cases.

The present study was done to estimate seropositivity of rickettsial infection in patients presenting with at least one week of undifferentiated febrile illness in Lady 
Hardinge Medical College, New Delhi. In this study, the mean age was 32.18 years. This correlates well with the active years of life

when people are mostly engaged in outdoor activities like farming and gardening. ${ }^{7}$

Table.1 Weil Felix agglutination titres in positive cases

\begin{tabular}{|l|l|l|l|}
\hline $\begin{array}{l}\text { Cases according to } \\
\text { titter value }\end{array}$ & $\begin{array}{l}\text { OXK } \\
\mathbf{1 0 0 \% ( 2 7 )}\end{array}$ & $\begin{array}{l}\text { OX19 } \\
\mathbf{1 0 0 \% ( 5 )}\end{array}$ & $\begin{array}{l}\text { OK2 } \\
\mathbf{1 0 0 \% ( 1 4 )}\end{array}$ \\
\hline$\geq \mathbf{1 : 8 0}$ & $51.9 \%(14)$ & $20 \%(1)$ & $42.9 \%(6)$ \\
\hline $\mathbf{1}: \mathbf{1 6 0}$ & $33.3 \%(9)$ & $80 \%(4)$ & $35.8 \%(5)$ \\
\hline$\geq \mathbf{1 : 3 2 0}$ & $11.1 \%(3)$ & - & $14.2 \%(2)$ \\
\hline$\geq \mathbf{1 : 6 4 0}$ & $3.7 \%(1)$ & - & $7.1 \%(1)$ \\
\hline
\end{tabular}

Fig.1 Age distribution of total 370 samples

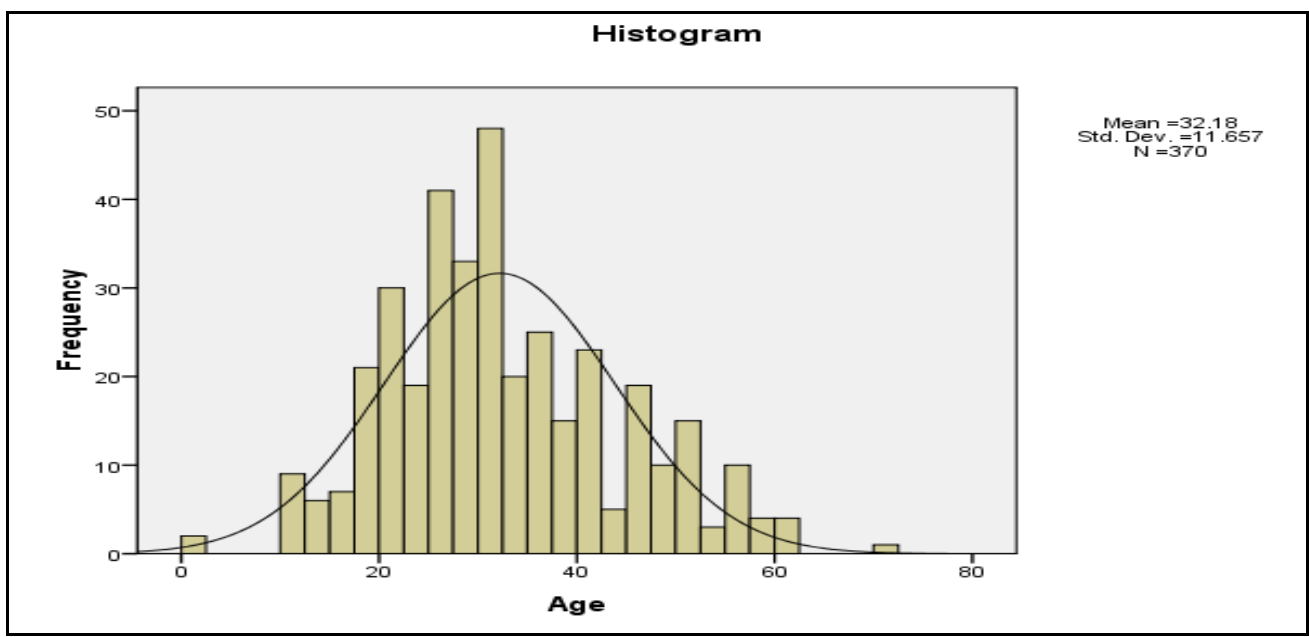

Fig.2 Frequency and distribution of duration of fever

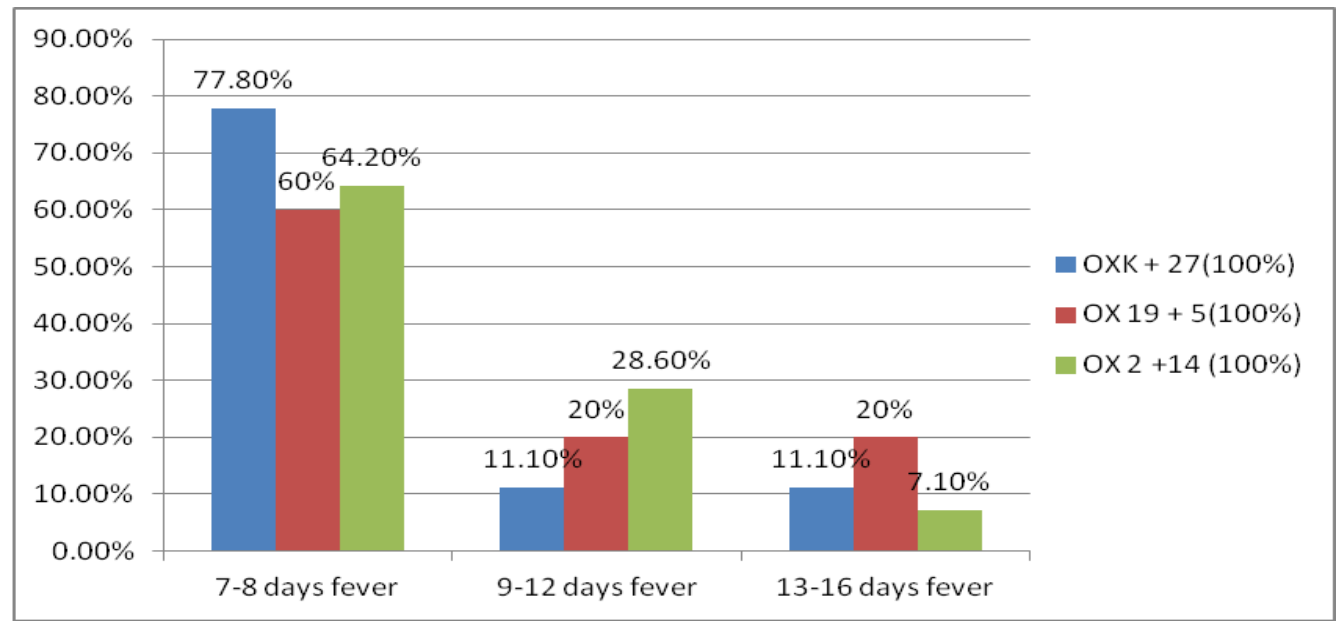


Fig.3 Distribution of clinical features among Weil Felix positive cases

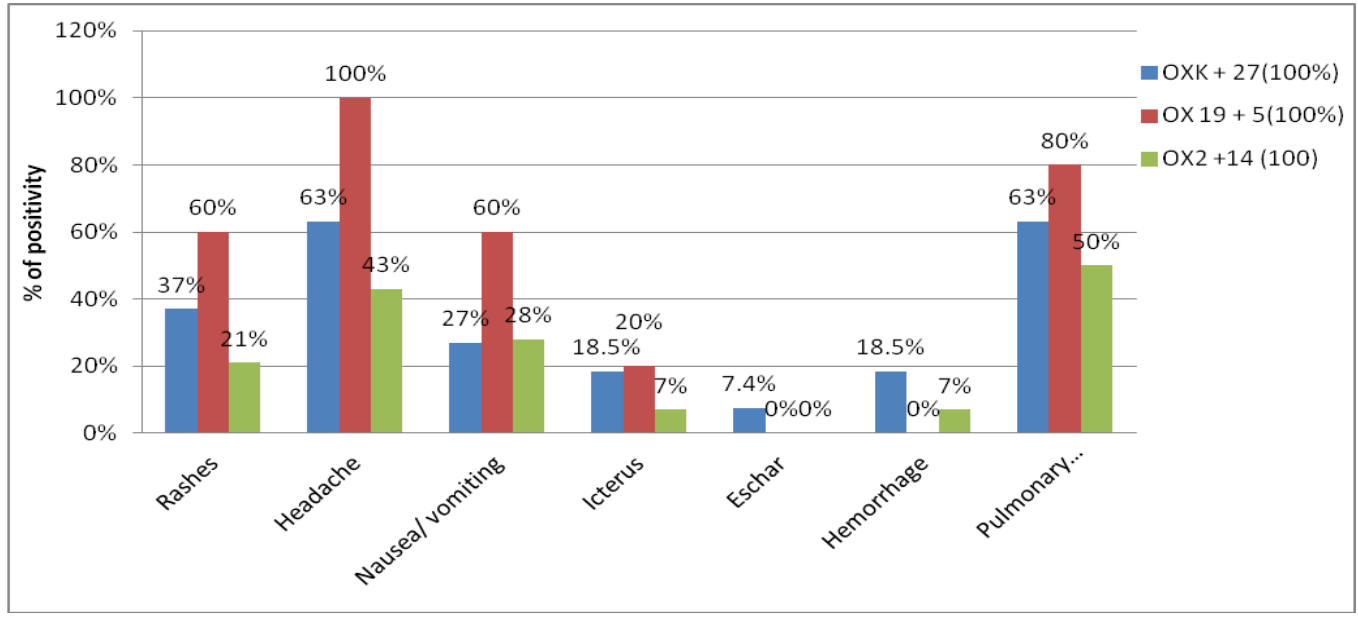

Fig.4 Laboratory findings among Weil- Felix positive cases

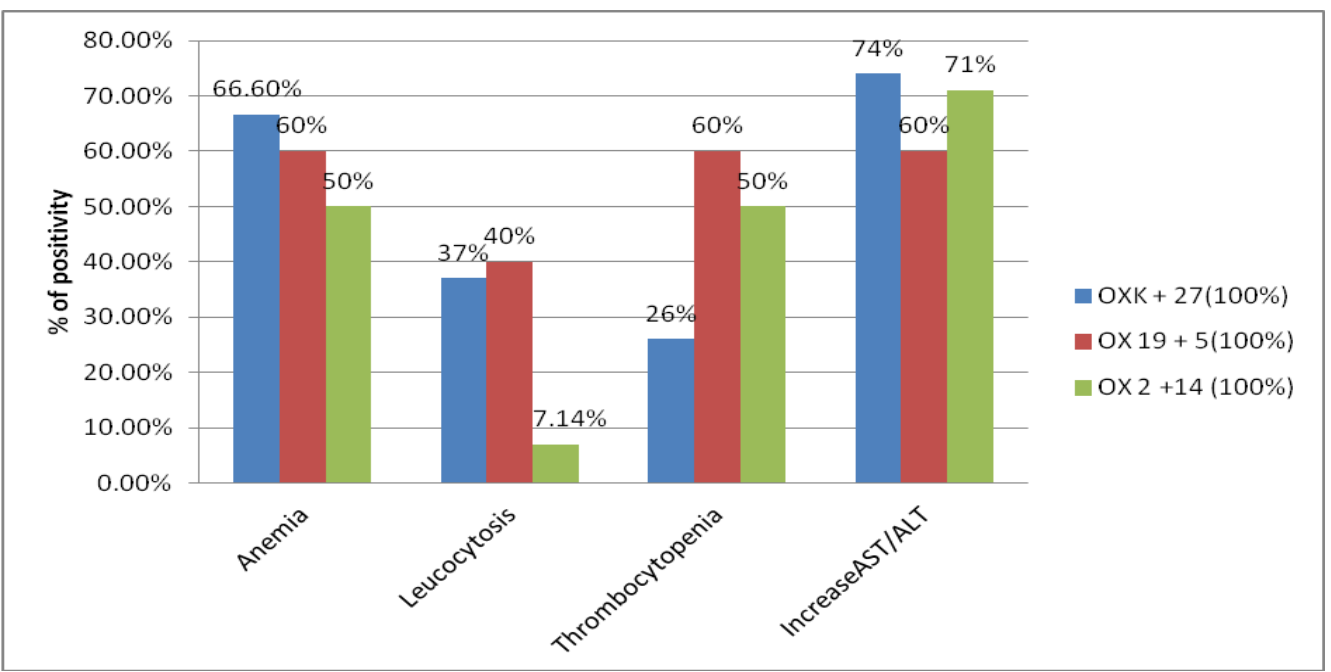

Chart.1 Distribution of patients according to geographical area

\section{Distribution of patients according to} geographical area.

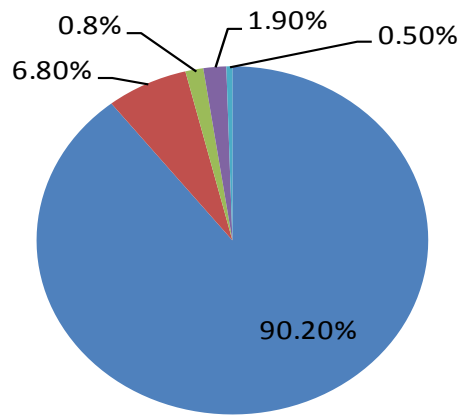

- New Delhi

- Utter Pradesh

- Punjab

- Haryana

Bihar 
Chart.2 Results of WFT

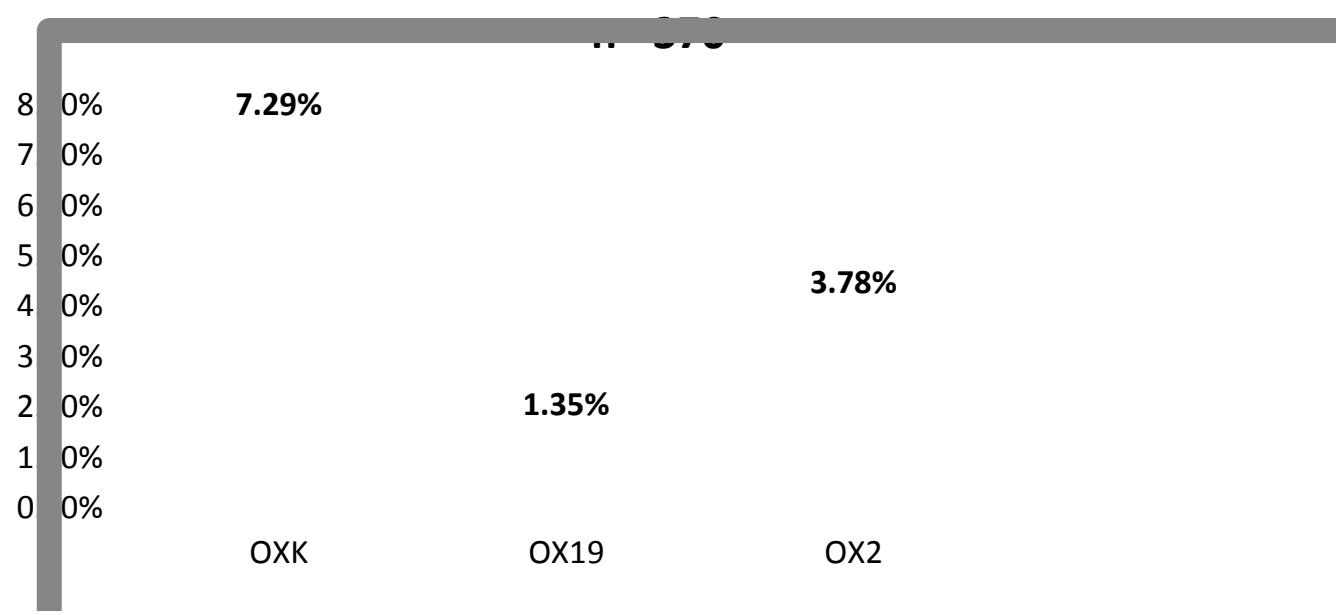

Most of the patients were females (61.6\%). No age and sex predilection has been seen for rickettsial infections. ${ }^{8}$ Ninety percent patients attending the hospital were residents of New Delhi area.

The review of published rickettsial outbreaks in the last decade indicates its continued presence, particularly scrub and tick typhus, in several parts of the India. These are being considered some of the most covert emerging and re-emerging diseases in India. ${ }^{2}$ However, due to its underreporting, the published literature is less likely to reflect the actual scenario of the disease in the country. ${ }^{8}$ Also, there is lack of awareness among clinicians. Due to outbreak of febrile illnesses such as dengue, chikungunya, typhoid, malaria, clinicians do not suspect rickettsial diseases. At present, Rickettsial infections in Delhi are rarely diagnosed because of its nonspecific clinical presentation and a low index of suspicion. Nevertheless, the findings of our study indicate the significant presence of rickettsial diseases in Delhi population.

In our prospective observational study conducted over 18 months, we used single acute phase sera from patients with undifferentiated febrile illness to determine antibodies against rickettsial diseases. By Weil Felix test, 7.29\% cases (27/370) were found positive for scrub typhus, $3.78 \%$ cases (14/370) were positive for tick typhus and $1.35 \%$ cases $(5 / 370)$ were positive for typhus group. In a study conducted by Mittal et al., in New Delhi in 2005-09 employing Weil Felix, reported seropositivity of scrub typhus as 3.5\%, tick typhus (OX2) as 3.2\% and typhus group (OX19) as $0.6 \%$. The same study reported scrub typhus in suspected cases to be around 16.05\%. ${ }^{6}$ Another study conducted by Gupta et al., in New Delhi in 2015 reported prevalence of scrub typhus as $14.4 \%$ and $13.57 \%$ by using immunofluorescence and $\operatorname{IgM}$ scrub typhus ELISA respectively. ${ }^{8}$ Other studies conducted in different parts of India reported prevalence rates ranging from $30.8 \%$ to $46 \% .^{8-10}$ The risk factors were changing clothes weekly or less frequently, tall grass in the neighbourhood, travel to the forest, bathing infrequency (less than thrice weekly), handling of tick-infested animals. ${ }^{2}$ Visits to rural and forest areas in endemic countries for activities such as camping, hiking, or rafting leads to travelacquired cases of scrub typhus. ${ }^{11}$ Earlier, the shrubs in hilly and forest terrains were thought to be the only habitat of the mite. But study conducted by Laskar et al., in 2015 
have shown that rodents carrying the mite are transmitting the disease in the urban locales as well. $^{12}$

In this study, the most common symptoms of scrub typhus were fever presenting with headache and pulmonary manifestations seen in $62.9 \%(17 / 27)$ of OXK positive cases. Rash was seen in only in $37 \%$ case, icterus in $18.5 \%$ and eschar in $7.4 \%(2 / 27)$ cases. This is in concordance with the result of study conducted by Gupta et al., in New Delhi where pulmonary manifestations were seen in $69.2 \%$ cases and rash was only seen in $24.2 \%$ (8/33) cases. Eschar and icterus was seen in only $18.2 \%$ and $15.2 \%$ cases respectively. ${ }^{8}$ Eschar if seen is pathognomic of scrub typhus. The low prevalence of eschar has been explained on the basis of its clandestine location because of which it is frequently missed on examination. Also, it is difficult to visualize on dark skinned individuals. ${ }^{8}$

Suggestive laboratory features includes normal to low leukocyte count with marked left shift, mildly elevated hepatic transaminases, hyponatremia and thrombocytopenia which points towards diagnosis of rickettsial disease, although absence of these does not rule it out. ${ }^{13}$ In our study, most common laboratory manifestation was increase in AST/ALT (70\%) followed by anaemia (66.4\%), leucocytosis (37\%) and thrombocytopenia (26\%).

Among typhus group positive cases, the most common symptom was fever associated with headache $(100 \%)$ followed by pulmonary manifestations $(80 \%)$. Common laboratory finding was anaemia (60\%), increase in AST/ALT (60\%) and thrombocytopenia $(60 \%)$. This result is in concordance with study conducted by Mittal et al., in New Delhi in where fever with rash $(51.1 \%)$ was the commonest presentation followed by headache $(41.4 \%)$. Common laboratory findings were thrombocytopenia $(44.8 \%)$ and increase in AST/ ALT (44.8\%). ${ }^{6}$ In tick typhus positive cases also, the most common symptom was pulmonary manifestations (50\%) followed by headache $(43 \%)$. Rashes were present only in $21 \%$ cases. Among the laboratory findings, increase in AST/ALT (71\%), anaemia (50\%) and thrombocytopenia $(50 \%)$ are most common features. Leucocytosis was seen only in one case (7.14\%). Similar results were seen in investigation done on rickettsial outbreaks. The common clinical presentation reported during the outbreaks reviewed included fever (>1 week) with chills and rigor, headache and vomiting. Rashes were a rare symptom. Abnormal kidney and liver function were the most common laboratory abnormalities observed. $^{2}$

Weil Felix test is the oldest assay based on detection of antibody to various Proteus antigens that cross-react with rickettsiae. In a study conducted by Farhana et al., the sensitivity and specificity of Weil Felix test; taking IgM ELISA as a reference standard were $75 \%$ and $79.9 \%$ respectively. ${ }^{1}$ Unfortunately, the laboratory tests that are more specific and sensitive than the commonly used Weil-Felix test such as the immunofluorescence assay (IFA), indirect immunoperoxidase (IP) test and the isolation of the organisms in animals or cell culture are not available and too expensive for most of the settings in India. ${ }^{2}$ Weil Felix test is cost effective, simple and easy to perform and is a good screening test for diagnosis of rickettsial infections. The use of Weil Felix test has to be interpreted in the correct clinical context but in conditions where definitive investigations are not possible its use is acceptable. $^{14}$

\section{Limitations of the study}

The limitations of this study were that current baseline titre for Weil Felix test were not established. Also, the confirmation of scrub 
typhus test by IFA or PCR was not performed. Also, in many cases the samples were collected at 7-8 days of febrile illness that could have lead to low seropositivity of these infections.

In conclusion, Rickettsial diseases are known to be the causes of acute febrile illness associated with life-threatening complications, if not diagnosed and treated on time. Due to the nonspecific symptoms and lack of awareness in most cases, diagnosis is difficult. Also the co-prevalence and similar presentation of other vector borne diseases like malaria, chikungunya and dengue lead to more confusion. By performing Weil-Felix test, we can simply get some information regarding the nature of infection, which can be confirmed by other techniques, if available. Clinicians should be encouraged to suspect rickettsial diseases in cases of acute undifferentiated febrile illnesses. Delhi hospitals as well as private laboratories should be encouraged to provide facilities to diagnose these diseases.

\section{Acknowledgement}

It gives me immense pleasure to express my thanks and gratitude to all those who enabled me in the successful completion of this study.

I am deeply indebted to my esteemed teacher and guide Dr. Manoj Jais, Director Professor, department of microbiology, LHMC, Delhi for his intellectual guidance and expert supervision throughout the course of this study.

I would like to express my heartfelt gratitude to Professor Dr. Ravinder Kaur, Head of Department of Microbiology, LHMC, Delhi, for allowing me to undertake the present study, for providing laboratory and other infrastructure to carry out this study.

My special thanks to Dr Harish K Pemde,
Professor, Department of Paediatrics, LHMC, Delhi, for his ardent interest, expert supervision and invaluable suggestions rendered in carrying out this study.

My heartfelt thanks to Dr. Anupam Prakash, Professor, Department of Medicine, LHMC, Delhi, for his meticulous evaluation and guidance throughout this study.

My special thanks to all the serology lab technicians for their generous help and kind support.

Most importantly, none of this would have been possible without love and patience of my family.

Words cannot adequately express the deep sense of gratitude I owe to all my seniors, colleagues and faculty members who cooperated with me and extended their kind help.

I express my deep sense of obligation to all my patients who made their invaluable contribution to the study.

I thank God, the almighty for the constant blessing, for providing me the capability to proceed successfully.

\section{References}

1. Farhana A, Bali N, Kanth F, Farooq R, Haq IU, Shah P. Serological Evidence of Scrub Typhus among Cases of PUO in the Kashmir Valley- A Hospital Based Study. J Clin Diagn Res JCDR. 2016 May; 10(5): DC24-DC26.

2. Dasari V, Kaur P, Murhekar MV. Rickettsial disease outbreaks in India: A review. Ann Trop Med Public Health. 2014 Nov 1; 7(6): 249.

3. Scrub typhus outbreak in Himachal affects $700, \quad$ kills 20. 
http://www.hindustantimes.com/. 2016

4. Rahi M, Gupte MD, Bhargava A, Varghese GM, Arora R. DHR-ICMR Guidelines for diagnosis \& management of Rickettsial diseases in India. Indian J Med Res. 2015 Apr 1; 141(4): 417.

5. Chauhan V, Rani S, Thakur K, Sood A, Chand Jaryal S, Sood A. Comparison of weil felix test and IgM ELISA in the diagnosis of scrub typhus in Kangra, Himachal Pradesh. Int J Health Sci Res. 2016 Nov 19; 6: 28-32.

6. Mittal V, Gupta N, Bhattacharya D, Kumar K, Ichhpujani RL, Singh S, et al., Serological evidence of rickettsial infections in Delhi. Indian J Med Res. 2012 Apr; 135(4): 538-41.

7. Sharma PK, Ramakrishnan R, Hutin YJF, Barui AK, Manickam P, Kakkar M, et al., Scrub typhus in Darjeeling, India: opportunities for simple, practical prevention measures. Trans R Soc Trop Med Hyg. 2009 Nov; 103(11): 1153-8.

8. Gupta N, Chaudhry R, Kabra SK, Lodha R, Mirdha BR, Das BK, et al., In Search of Scrub Typhus: A Prospective Analysis of Clinical and Epidemiological Profile of Patients from a Tertiary Care Hospital in New Delhi. Adv Infect Dis. 2015 Oct
20; 5(4): 140.

9. Sharma A, Kanga A, Sharma V, Mahajan S, M. L. Gupta. Investigation of an Outbreak of Scrub Typhus in the Himalayan Region of India. Jpn J Infect Dis. 2005 Aug; 58(4): 208-10

10. Mahajan SK, Rolain J-M, Kashyap R, Bakshi D, Sharma V, Prasher BS, et al., Scrub Typhus in Himalayas. Emerg Infect Dis. 2006 Oct; 12(10): 1590-2.

11. Nicholson W, Paddock C. Rickettsial (Spotted \& Typhus Fevers) \& Related Infections, including Anaplasmosis \& Ehrlichiosis. In: Yellow Book- Centers for Disease Control and Prevention. 2017.

12. Laskar AR, Suri S, Acharya AS. Scrub Typhus: Re-emerging Public Health Problem in India. J Commun Dis. 2015 Sep 30; 47(3): 19-25.

13. Rathi N, Rathi A. Rickettsial infections: Indian perspective. Indian Pediatr. 2010 Feb; 47(2): 157-64.

14. Mahajan SK, Kashyap R, Kanga A, Sharma V, Prasher BS, Pal LS. Relevance of Weil-Felix test in diagnosis of scrub typhus in India. J. Assoc Physicians India. 2006 Aug; 54: 619-21.

\section{How to cite this article:}

Garima Gautam, Manoj Jais, Anupam Prakash and Harish K Pemde. 2019. Emerging Rickettsial Diseases: an Analysis of Undifferentiated Acute Febrile Illness Cases from a Tertiary Care Hospital in New Delhi. Int.J.Curr.Microbiol.App.Sci. 8(03): 463-471. doi: https://doi.org/10.20546/ijcmas.2019.803.058 